\title{
Muon-Fluorine Entangled States in Molecular Magnets
}

\author{
T. Lancaster, ${ }^{1 *}$ S. J. Blundell, ${ }^{1}$ P. J. Baker, ${ }^{1}$ M. L. Brooks, ${ }^{1}$ W. Hayes, ${ }^{1}$ F. L. Pratt, ${ }^{2}$ J. L. Manson, ${ }^{3}$ \\ M. M. Conner, ${ }^{3}$ and J. A. Schlueter ${ }^{4}$ \\ ${ }^{1}$ Clarendon Laboratory, Department of Physics, Oxford University, Parks Road, Oxford, OX1 3PU, United Kingdom \\ ${ }^{2}$ ISIS Facility, Rutherford Appleton Laboratory, Chilton, Oxfordshire OX11 OQX, United Kingdom \\ ${ }^{3}$ Department of Chemistry and Biochemistry, Eastern Washington University, Cheney, Washington 99004, USA \\ ${ }^{4}$ Material Science Division, Argonne National Laboratory, Argonne, Illinois 60439, USA
}

(Received 18 July 2007; published 27 December 2007)

\begin{abstract}
The information accessible from a muon-spin relaxation experiment can be limited due to a lack of knowledge of the precise muon stopping site. We demonstrate here the possibility of localizing a spin polarized muon in a known stopping state in a molecular material containing fluorine. The muon-spin precession that results from the entangled nature of the muon spin and surrounding nuclear spins is sensitive to the nature of the stopping site. We use this property to identify three classes of sites that occur in molecular magnets and describe the extent to which the muon distorts its surroundings.
\end{abstract}

DOI: 10.1103/PhysRevLett.99.267601

Muon-spin relaxation $\left(\mu^{+} \mathrm{SR}\right)$ continues to provide insights into the nature of magnetic materials, superconductors, semiconductors, soft matter, and chemical reactions [1]. The technique involves stopping spin polarized muons in a sample where the muons probe the distribution of local magnetic fields across the muon stopping sites. Despite its successes, two concerns are sometimes raised. The first is that the exact muon stopping site in a material is, in general, not precisely known, introducing some uncertainty in the determination of the local spin structure. A second concern is that it is not generally possible to quantify the degree to which the presence of the charged muon distorts its surroundings and hence to estimate the modification of superexchange pathways that could, in principle, affect the local spin structure. Here we show that, in addition to the magnetic behavior usually probed by $\mu^{+} \mathrm{SR}$, the entangled states of the $\mu^{+}$spin and ${ }^{19} \mathrm{~F}$ nuclear spins allow the accurate determination of the muon site in a class of novel fluorinated molecular magnets, along with an estimate of the distortion introduced by the presence of the probe particle.

Localized muons often interact with their environment via dipole-dipole coupling. In many cases the large number of spin centers surrounding the muon allows the use of the local magnetic field (LMF) approximation, where the muon spin $S$ interacts with the net local magnetic field at the $i$ th muon site $\langle\boldsymbol{B}\rangle_{i}$ via the Hamiltonian $\mathcal{H}=\gamma_{\mu} \boldsymbol{S}$. $\langle\boldsymbol{B}\rangle_{i}$, where $\gamma_{\mu}\left(=2 \pi \times 135.5 \mathrm{MHzT}^{-1}\right)$ is the muon gyromagnetic ratio. For the commonly encountered case of a muon ensemble interacting with an array of randomized static local fields, the LMF model gives the KuboToyabe (KT) muon polarization function [2]. Occasionally, however, the muon is found to be dipole coupled to a small number of spin centers $[3,4]$, with the resulting entangled spin states evolving via the Hamiltonian

$$
\mathcal{H}=\sum_{i>j} \frac{\mu_{0} \gamma_{i} \gamma_{j}}{4 \pi|\boldsymbol{r}|^{3}}\left[\boldsymbol{S}_{i} \cdot \boldsymbol{S}_{j}-3\left(\boldsymbol{S}_{i} \cdot \hat{\boldsymbol{r}}\right)\left(\boldsymbol{S}_{j} \cdot \hat{\boldsymbol{r}}\right)\right],
$$

PACS numbers: 76.75.+i, 61.05.Qr, 75.50.Xx

where $\boldsymbol{r}$ is the vector linking spins $\boldsymbol{S}_{i}$ and $\boldsymbol{S}_{j}$, which have gyromagnetic ratios $\gamma_{i, j}$. This strong interaction is found most often in materials containing fluorine, for two reasons: (1) fluorine is the most electronegative element, causing muons to preferentially localize in its vicinity; (2) fluorine occurs with a single isotope $\left({ }^{19} \mathrm{~F}\right)$ with an $I=$ $1 / 2$ nuclear spin. A well-known case of entanglement occurs in most insulating metal fluorides [5] where the muon sits midway between two $\mathrm{F}^{-}$ions forming a strong linear "hydrogen bond" with an F-F separation of $d=$ $0.238 \mathrm{~nm}$ (approximately twice the fluorine ionic radius). This so-called $\mathrm{F}-\mu^{+}-\mathrm{F}$ state is similar to the $V_{k}$ center observed in alkali halides, which is often treated as a molecule-in-a-crystal defect [6], where the host weakly perturbs the molecular ion.

These considerations suggest that in compounds containing fluorine a significant fraction of muons may be localized at positions near fluorine nuclei. The $\mu^{+}$and ${ }^{19} \mathrm{~F}$ spins become entangled, causing the spins to evolve via the Hamiltonian in Eq. (1) and the sensitivity of the interaction to the relative positions of the spin centers allows the stopping site to be determined. This is indeed the case in a novel class of fluorine-containing molecular magnets that we consider here. The stopping states are, however, more complex than the linear $\mathrm{F}-\mu^{+}-\mathrm{F}$ molecular ion previously observed.

In this Letter we consider three molecular antiferromagnets (AFMs), which are formed through bridging paramagnetic $\mathrm{Cu}^{2+}$ centers with ligands such as pyrazine (pyz) (see Table I). Below an antiferromagnetic transition at $T_{N}$ we observe oscillations in the time dependence of the muon polarization [the "asymmetry" $A(t)$ [1]] which are characteristic of a quasistatic local magnetic field at the muon stopping site. In the LMF model, this causes a coherent precession (at a frequency $\omega_{i}=\gamma_{\mu}\left|\langle\boldsymbol{B}\rangle_{i}\right|$ ) of the spins of those muons which have a component of their spin polarization perpendicular to the local field. Above $T_{N}$ the character of the measured spectra changes considerably 
TABLE I. Parameters for Eq. (3) for each material studied with resulting $\chi^{2}$ for the fits described in the main text. $\chi_{\mathrm{F} \mu \mathrm{F}}^{2}$ corresponds to the best fits obtained using a conventional $\mathrm{F}-\mu^{+}-\mathrm{F}$ model for comparison.

\begin{tabular}{lcccccc}
\hline \hline & $p_{1}$ & $\lambda(\mathrm{MHz})$ & $p_{2}$ & $\sigma(\mathrm{MHz})$ & $\chi^{2}$ & $\chi_{\mathrm{F} \mu \mathrm{F}}^{2}$ \\
\hline $\mathrm{CuF}_{2}\left(\mathrm{H}_{2} \mathrm{O}\right)_{2}(\mathrm{pyz})$ & 0.43 & 0.36 & 0.57 & 0.56 & 1.2 & 1.6 \\
$\mathrm{CuNO}_{3}(\mathrm{pyz})_{2} \mathrm{PF}_{6}$ & 0.65 & 0.24 & 0.35 & 0.45 & 2.2 & 4.1 \\
{$\left[\mathrm{Cu}\left(\mathrm{HF}_{2}\right)\left(\mathrm{pyz}_{2}\right] \mathrm{ClO}_{4}\right.$} & 0.73 & 0.24 & 0.27 & 0.43 & 2.2 & 3.9 \\
\hline \hline
\end{tabular}

and we observe lower frequency oscillations characteristic of the dipole interaction of the entangled muon and the ${ }^{19} \mathrm{~F}$ spins. This is the case because the $\mathrm{Cu}^{2+}$ electronic moments, which dominate the spectra for $T<T_{N}$, fluctuate very rapidly on the muon time scale for $T>T_{N}$ and are therefore motionally narrowed from the spectra, leaving the muon sensitive to the quasistatic nuclear magnetic moments. Generally, the initial spin state is not an eigenstate of Eq. (1). This causes a time dependence in the system's total wave function, visualized classically as a spontaneous precession of all spin species. The observed property in a $\mu^{+} \mathrm{SR}$ experiment is the polarization $D_{z}(t)$ of the muon ensemble along the initial muon-spin direction $z$, which, for the powder samples considered here, is given by [7]

$$
D_{z}(t)=\frac{1}{N}\left\langle\sum_{m, n}\left|\left\langle m\left|\sigma_{q}\right| n\right\rangle\right|^{2} \exp \left(\mathrm{i} \omega_{m n} t\right)\right\rangle_{q}
$$

where $N$ is the number of spins, $|m\rangle$ and $|n\rangle$ are eigenstates of the total Hamiltonian $\mathcal{H}, \sigma_{q}$ is the Pauli spin matrix corresponding to the direction $q$ and \langle\rangle$_{q}$ represents an appropriately weighted powder average over all directions. The signal arising from $\mathrm{F}-\mu^{+}$states persists in these materials to temperatures well above $100 \mathrm{~K}$. These oscillating components are not observed for similar molecular magnets that do not contain fluorine.

Above $T_{N}$, where the muon spin is relaxed through interaction with nuclear moments, we expect two contributions to the $\mu^{+} \mathrm{SR}$ spectra. The first is from muons strongly coupled to fluorine nuclei, which give rise to contributions derived from Eq. (2). The second arises either from the random fields due to more distant nuclear moments or possibly from alternative muon sites not strongly coupled to a fluorine nucleus, and it is expected to give rise to a KT function. By analogy with previous results on similar, but unfluorinated, systems [8], we note that the full KT function is not usually observed. Instead a Gaussian function $\exp \left(-\sigma^{2} t^{2}\right)$, often used to approximate the KT function in the presence of slow dynamics, successfully accounts for the measured spectra. Such a function is used here. Above the critical temperature $T_{N}$, spectra were found to be well described by the resulting polarization function

$$
A(t)=A_{0}\left[p_{1} D_{z}(t) \exp (-\lambda t)+p_{2} \exp \left(-\sigma^{2} t^{2}\right)\right]+A_{\mathrm{bg}}
$$

where $A_{0}$ is the signal arising from the sample, $p_{1}+p_{2}=$ 1 , and $A_{\mathrm{bg}}$ accounts for those muons that stop in the sample holder or cryostat tails. Below we discuss three distinct classes of implanted muon states observed in molecular magnets [9].

Case I: Interaction with a single fluorine. - The compound $\mathrm{CuF}_{2}\left(\mathrm{H}_{2} \mathrm{O}\right)_{2}$ (pyz) [10] is formed from $\mathrm{CuF}_{2} \mathrm{O}_{2} \mathrm{~N}_{2}$ octahedra linked with pyrazine bridges along the $a$ direction forming linear chains [Fig. 1(a)]. Extensive hydrogen bonding interactions tether the chains into the 3D network shown in Fig. 1(b). The material undergoes an AFM transition at $T_{N}=2.54(8) \mathrm{K}$ [10]. Zero field (ZF) $\mu^{+} \mathrm{SR}$ spectra measured on $\mathrm{CuF}_{2}\left(\mathrm{H}_{2} \mathrm{O}\right)_{2}\left(\right.$ pyz) above $T_{N}$ are shown in Fig. 1(c) where we see oscillations characteristic of $\mathrm{F}-\mu^{+}$dipole coupled states. The spectra are most successfully modeled by assuming the muon is strongly coupled with a single $I=\frac{1}{2} \mathrm{~F}$ spin, localized a distance $d=$ $0.110(2) \mathrm{nm}$ away from a fluorine nucleus. This compares with a $\mathrm{F}-\mu^{+}$distance of $0.119(1) \mathrm{nm}$ in the conventional $\mathrm{F}-\mu^{+}-\mathrm{F}$ case [5]. The resulting energy level structure for
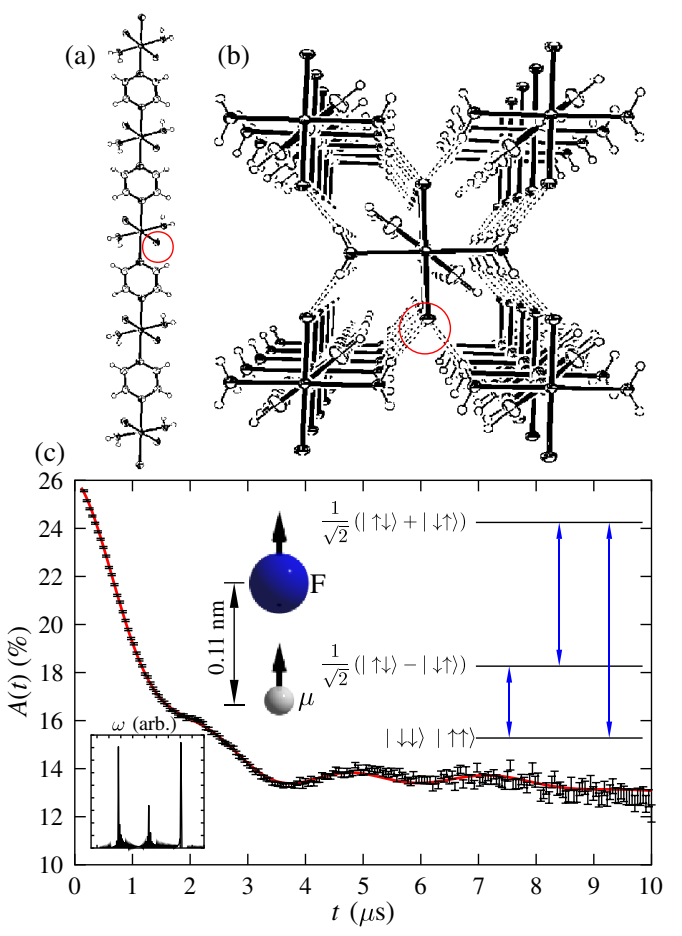

FIG. 1 (color online). (a) Linear chain structure of $\mathrm{CuF}_{2}\left(\mathrm{H}_{2} \mathrm{O}\right)_{2}(\mathrm{pyz})$. The circled regions show sample fluorine atoms near which muons are expected to localize. (b) Crystal packing viewed parallel to the chain axis. Dotted lines show $\mathrm{OH} \cdot \mathrm{F}$ hydrogen bonds. (c) Example ZF $\mu^{+}$SR spectrum measured at $T=3.4 \mathrm{~K}$ with a fit to the $\mathrm{F}-\mu^{+}$dipole interaction described in the text. Inset: $\mathrm{F}-\mu^{+}$configuration: the resulting energy levels (top right) with allowed transitions and the calculated frequency spectrum (bottom left). 
this scenario is shown in the inset in Fig. 1(c). The F- $\mu^{+}$ spin system consists of three distinct energy levels with three expected transitions between them giving rise to the distinctive three-frequency oscillations observed [Fig. 1(c) inset]. The signal is described by a polarization function $D_{z}(t)=\frac{1}{6}\left[1+\sum_{j=1}^{3} u_{j} \cos \left(\omega_{j} t\right)\right]$, where $u_{1}=2, u_{2}=1$, and $u_{3}=2$. The transition frequencies [shown in Fig. 1(c)] are given by $\omega_{j}=j \omega_{d} / 2$, where $\omega_{d}=$ $\mu_{0} \gamma_{\mu} \gamma_{\mathrm{F}} / 4 \pi r^{3}$, and $r$ is the $\mathrm{F}-\mu^{+}$separation. The resulting fit is shown in Fig. 1(c) using the parameters listed in Table I. The muon-fluorine distance was also varied as a parameter in the fitting routine.

The strong interaction of the muon and a single $F$ is unusual. However, a muon stopping site between two fluorines is probably made energetically unfavorable due to the presence of the protons on the $\mathrm{H}_{2} \mathrm{O}$ groups which are hydrogen bonded to the fluorines. In this material the smallest F-F distance (between adjacent chains) is $0.34 \mathrm{~nm}$ and a position midway between these two fluorines invariably lies very close $(\sim 0.13 \mathrm{~nm})$ to the protons on a $\mathrm{H}_{2} \mathrm{O}$ group. It is probable that the muon's separation from the $\mathrm{F}$ ion is not precisely in the $a-b$ plane, but rather has a small component in the $c$ direction, taking it closer to the electron density on the aromatic rings. We note further that we have also observed coupling of the muon to a single $\mathrm{F}$ in the polymer PVDF $\left[-\left(\mathrm{CH}_{2} \mathrm{CF}_{2}\right)_{n}-\right]$ below the glass transition [11], demonstrating that this stopping state is not unique to this material. Previous work on muon stopping states suggested that possible muon sites may involve muon addition to the pyrazine rings [12] or muon substitution of a proton in the $\mathrm{H}_{2} \mathrm{O}$ groups [13]. The dipole coupling to fluorine nuclei at these candidate sites cannot account for the oscillating fraction of the signal. It is possible, however, that occupancies at these sites contribute to the fraction of the signal with amplitude $p_{2}$.

Case II: Crooked $F-F$ bond with the $\mathrm{PF}_{6}{ }^{-}$ion. - The quasi 2D compound $\left[\mathrm{Cu}\left(\mathrm{NO}_{3}\right)(\mathrm{pyz})_{2}\right] \mathrm{PF}_{6}[14,15]$ is formed from infinite $2 \mathrm{D}$ sheets of $\left[\mathrm{Cu}(\mathrm{pyz})_{2}\right]^{2+}$ lying in the $a b$ plane. These are linked along the $c$ direction by $\mathrm{NO}_{3}{ }^{-}$ions. The $\mathrm{PF}_{6}{ }^{-}$anions occupy the body-centered positions within the pores. The $\mathrm{PF}_{6}{ }^{-}$anion has a regular octahedral geometry with a P-F distance of $0.157 \mathrm{~nm}$. Our measurements show that this material magnetically orders below $T_{N}=2.0(2) \mathrm{K}$. Above $T_{N}$ we again observe a signal from dipole coupling. An example spectrum measured above $T_{N}$ is shown in Fig. 2. While one might expect the muon to localize near a $\mathrm{PF}_{6}{ }^{-}$anion, it is not clear a priori how many fluorine centers will be strongly coupled to the muon spin. Modeling the spectra reveals that two fluorine spins interact with the $\mu^{+}$, but not via the linear $\mathrm{F}-\mu^{+}-\mathrm{F}$. Instead we find the $\mathrm{F}-\mu^{+}-\mathrm{F}$ bond angle to be $\theta=143(1)^{\circ}$ with $\mathrm{F}-\mu^{+}$lengths $d_{1}=0.106(3) \mathrm{nm}$ and $d_{2}=$ $0.156(3) \mathrm{nm}$. The F-F distance, which is $0.22 \mathrm{~nm}$ in the unperturbed material, lengthens to $0.25 \mathrm{~nm}$. This configuration is shown in the inset in Fig. 2. A fit to Eq. (3) resulted

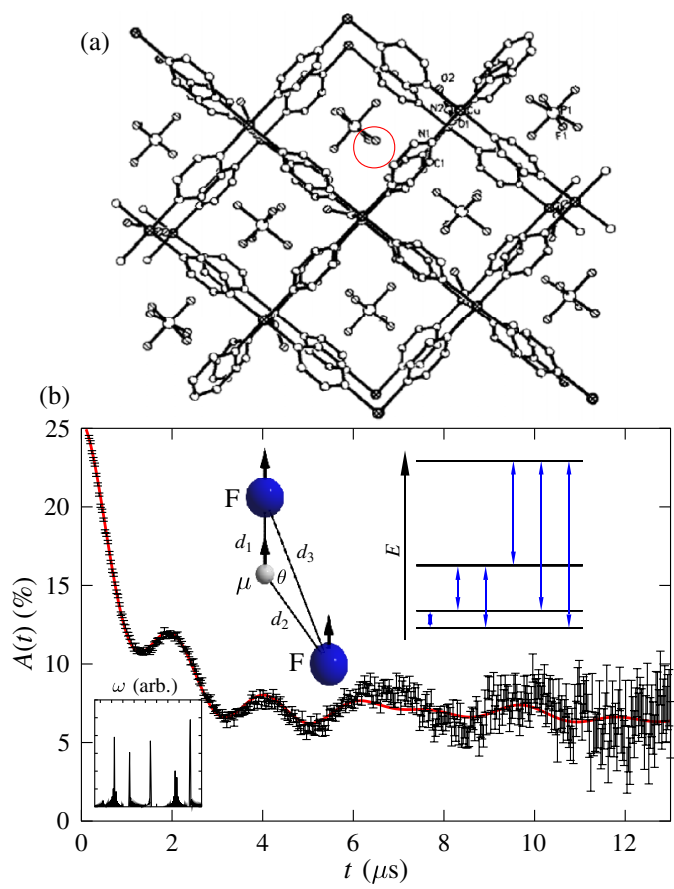

FIG. 2 (color online). (a) Structure of $\left[\mathrm{Cu}\left(\mathrm{NO}_{3}\right)\left(\mathrm{pyz}_{2}\right] \mathrm{PF}_{6}\right.$ in the $a-b$ plane showing $2 \mathrm{D}$ sheets of $\left[\mathrm{Cu}(\mathrm{pyz})_{2}\right]^{2+}$ and $\mathrm{PF}_{6}{ }^{-}$ anions occupying the pores in the structure [14]. The circled region shows sample fluorines near which the muon is expected to localize. (b) Example ZF spectra measured at $T=5.7 \mathrm{~K}$ with a fit described in the text. Inset: proposed $\mathrm{F}-\mu^{+}$configuration: (top right) the resulting energy levels with allowed transitions and (bottom left) the calculated frequency spectrum.

in the parameters listed in Table I and is shown in Fig. 2 (in the fitting routine the muon was fixed at the origin and the positions of $2 \mathrm{~F}$ nuclei were varied in two dimensions). It may also be expected that other materials containing $X_{\mathrm{F}_{6}}{ }^{-}$ ions $(X=\mathrm{Sb}$ or As) will have stopping states of this type. As before, we also note that the fluorine coupling from positions near the electronegative $\mathrm{NO}_{3}{ }^{-}$ions or the pyrazine rings is not sufficient to describe the oscillations, but these sites may account for some of the amplitude $p_{2}$.

Case III: Interaction with the $\mathrm{HF}_{2}{ }^{-}$ion. - The series of coordination polymers $\left[\mathrm{Cu}\left(\mathrm{HF}_{2}\right)(\mathrm{pyz})_{2}\right] Y$ are formed from infinite 2D sheets of $\left[\mathrm{Cu}(\mathrm{pyz})_{2}\right]^{2+}$ in the $a b$ plane [as in the case of $\left[\mathrm{Cu}\left(\mathrm{NO}_{3}\right)(\mathrm{pyz})_{2}\right] \mathrm{PF}_{6}$ above]. These are connected along the $c$ axis by linear $\mathrm{HF}_{2}{ }^{-}$anions to form a pseudocubic network. Small tetrahedral or octahedral anions $Y$ occupy the body centered positions in the pseudocubic pores [16]. An example spectrum measured above $T_{N}=$ $1.94 \mathrm{~K}$ for $\left[\mathrm{Cu}\left(\mathrm{HF}_{2}\right)(\text { pyz })_{2}\right] \mathrm{ClO}_{4}$ is shown in Fig. 3(b). In this case we might expect the muon to localize near the $\mathrm{HF}_{2}{ }^{-}$anion. The spectrum obtained is qualitatively similar to that expected for the interaction of a muon with a single fluorine ion. However, the best fit is obtained if a third spin center is included. For the tightly bound $\mathrm{HF}_{2}{ }^{-}$ion we might expect this third center to be that of the nearest proton [see Fig. 3(b) inset]. This configuration is found 


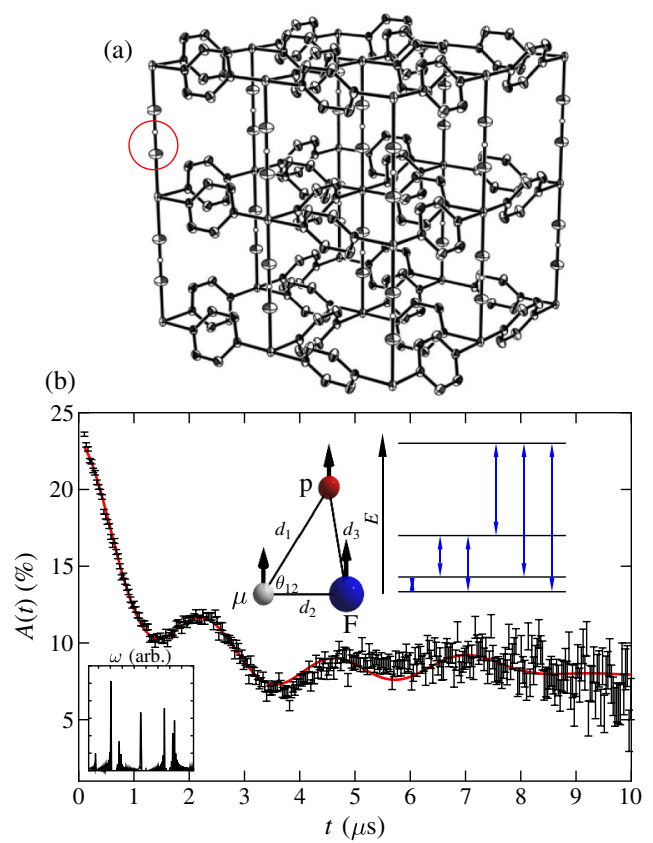

FIG. 3 (color online). (a) Structure of $\left[\mathrm{Cu}\left(\mathrm{HF}_{2}\right)(\mathrm{pyz})_{2}\right] Y$, with $Y$ molecules omitted for clarity. The circled region shows a sample fluorine and proton near which the muon is expected to localize. (b) Example ZF spectra measured at $T=11 \mathrm{~K}$ with a fit described in the text. Inset: proposed $\mathrm{F}-\mu^{+}-p$ configuration: (top right) the resulting energy levels with allowed transitions and (bottom left) the calculated frequency spectrum.

to fit the data successfully yielding a $\mathrm{F}-\mu^{+}$distance of $d_{1}=0.111(3) \mathrm{nm}$, a $\mu^{+}-p$ distance of $d_{2}=0.161(3) \mathrm{nm}$, and a $\mathrm{F}-\mu^{+}-p$ angle of $\theta=57(1)^{\circ}$. The $p-\mathrm{F}$ distance $d_{3}$, which is $0.11 \mathrm{~nm}$ in the unperturbed system, is found to be increased to $d_{3}=0.137(3) \mathrm{nm}$ in the presence of the muon. The resulting fit to Eq. (3) results in the fitting parameters given in Table I and is shown in Fig. 3(b) (the muon was fixed at the origin in the fitting routine, with the positions of a fluorine spin and proton spin varied in two dimensions). Candidate sites that do not give rise to the oscillations observed, but may contribute to $p_{2}$, again include muon addition to the pyz rings and also sites near the $\mathrm{ClO}_{4}{ }^{-}$groups. We note that our suggested configuration differs from that proposed for the HF dimer on the basis of intermolecular perturbation calculations [17]. The difference may be attributed to the presence, in the present case, of $\mathrm{Cu}^{2+}$ ions at either ends of the linear $\mathrm{HF}_{2}{ }^{-}$ groups. These will act to raise the potential of a positive muon in the vicinity of the position suggested by the HF dimer calculations.

For the conventional $\mathrm{F}-\mu^{+}-\mathrm{F}$ state the $\mathrm{F}$ atoms may each shift by large distances $(\sim 1 \AA)$ from their equilibrium positions toward the $\mu^{+}$[5], demonstrating that the muon introduces a non-negligible local distortion in the material. If, however, by analogy with the $V_{k}$ defect center in alkali halides [6], the $\mathrm{F}-\mu^{+}-\mathrm{F}$ complex acts like an independent molecule in the crystal, the distortion in the other ion positions will be much less significant than the distortion of the two $\mathrm{F}^{-}$ions. The $V_{k}$ center analogy will be less apposite in cases I-III described above, where the nonlinear bonds demonstrate that the muon stopping state cannot be regarded as separate from its surroundings. In these cases the tightly bound nature of the fluorinecontaining complexes prevents an independent quasimolecular impurity from forming. We note finally that our results demonstrate that the introduction of fluorine ions in molecular magnets can provide "traps" for muons, so that the local spin structure in such systems can be probed from well characterized muon sites.

This work is supported by the EPSRC (U.K.). T.L. acknowledges support from the Royal Commission for the Exhibition of 1851. J.L.M. acknowledges support from Research Corporation. Work at Argonne National Laboratory is sponsored by the U.S. Department of Energy, Office of Basic Energy Sciences, Division of Materials Sciences, under Contract No. DE-AC0206CH11357. We thank A.M. Stoneham for useful discussions.

*t.lancaster1@physics.ox.ac.uk

[1] S. J. Blundell, Contemp. Phys. 40, 175 (1999).

[2] R. S. Hayano et al., Phys. Rev. B 20, 850 (1979).

[3] J.S. Lord, S.P. Cottrell, and W. G. Williams, Physica (Amsterdam) 289B, 495 (2000).

[4] M. Celio and P. F. Meier, Hyperfine Interact. 17-19, 435 (1984).

[5] J.H. Brewer et al., Phys. Rev. B 33, 7813 (1986).

[6] W. Hayes and A.M. Stoneham, Defects and Defect Processes in Nonmetallic Solids (Dover, New York, 2004).

[7] E. Roduner and H. Fischer, Chem. Phys. 54, 261 (1981).

[8] T. Lancaster et al., Phys. Rev. B 73, 020410(R) (2006).

[9] Zero field $\mu^{+}$SR measurements were made on the MuSR instrument at the ISIS Facility, Rutherford Appleton Laboratory, U.K. Powder samples were packed in $25 \mu \mathrm{m} \mathrm{Ag}$ foil and mounted on an Ag backing plate inside an Oxford Instruments sorption cryostat.

[10] J. L. Manson et al. (unpublished).

[11] T. Lancaster et al. (unpublished).

[12] Z. Wu et al., J. Am. Chem. Soc. 113, 9096 (1991).

[13] A. Schenck and K. M. Crowe, Phys. Rev. Lett. 26, 57 (1971).

[14] M. M. Turnbull et al., Mol. Cryst. Liq. Cryst. 335, 245 (1999).

[15] F. M. Woodward et al., Inorg. Chem. 46, 4256 (2007).

[16] J. L. Manson et al., Chem. Commun. (Cambridge) 2006, 4894 (2006).

[17] M. P. Hodges, A. J. Stone, and E. C. Lago, J. Phys. Chem. A 102, 2455 (1998). 